EGU2020-18259

https://doi.org/10.5194/egusphere-egu2020-18259

EGU General Assembly 2020

(c) Author(s) 2021. This work is distributed under

the Creative Commons Attribution 4.0 License.

\title{
Validation of IVINE crop growth model using MACSUR2 project measurements in a few European vineyards.
}

\author{
Claudio Cassardo ${ }^{1,2}$, Valentina Andreoli ${ }^{3}$, and Federico Spanna ${ }^{4}$ \\ ${ }^{1}$ Univ. of Torino, Dept. of Physics and Natrisk center, Physics, Torino, Italy (claudio.cassardo@unito.it) \\ ${ }^{2}$ Ewha Womans University, College of Environmental Science and Engineering, Seoul, Republic of Korea \\ (claudio.cassardo@unito.it) \\ ${ }^{3}$ Univ. of Torino, Dept. of Physics and Natrisk center, Physics, Torino, Italy (valentina.andreoli@unito.it) \\ ${ }^{4}$ Phytosanitary sector, Regione Piemonte, Torino, Italy (federico.spanna@regione.piemonte.it)
}

The numerical crop growth model IVINE (Italian Vineyard Integrated Numerical model for Estimating physiological values) was originally developed at the dept. of Physics, Univ. of Torino, as a research model with the aim to simulate grapevine phenological and physiological processes. Since vines are generally strongly sensitive to meteorological conditions, the model should be able to evaluate the environmental forcing effects on vine growth and, eventually, on its production. IVINE model requires a set of hourly meteorological and soil data as boundary conditions; the more relevant input for the model to correctly simulate the plant growth are: air temperature and soil moisture. Among the principal IVINE outputs, we mention: the main philological stages (dormancy exit, bud-break, fruit set, veraison, and harvest), the leaf development, the yield, the berry sugar concentration, and the predawn leaf water potential. The IVINE requires to set some experimental parameters depending on the cultivar; at present, IVINE is optimized for Nebbiolo and other common varieties (such as, for example, cvs. Barbera, Vermentino, Cannonau, etc for Italy), but validation experiments have been performed only for Nebbiolo variety, due to the difficulty to gather all required measurements useful to drive the model and to compare its outputs for several consecutive years in the same vineyard. In the frame of the second part of the EU JPI-FACCE project named MACSUR (Modelling European Agriculture with Climate Change for Food Security), some data relative to vineyards displaced in several European countries were made available, thus we tried to execute simulations with IVINE in those vineyards. Since input data required by IVINE were not all present, we decided to extract input data from the international GLDAS database in the nearest grid point to the experimental vineyard, and to run the trusted land surface model UTOPIA on those points in order to evaluate soil variables required by IVINE. The main results obtained by those simulations, as well as the few possible validations with experimental observations, will be shown and commented. As a summary, we can say that the simulation carried out with IVINE seems able to well account for the interannual variability of the meteorological conditions, and the used settings seems able to allow a sufficiently valid simulation of the pheno-physiological conditions of the vineyards, but the approximation in the input data causes departures larger than if local measurements would be used. 
